\title{
EXPERIMENTAL AND THEORETICAL STUDIES OF EARLY TURBULENCE*
}

\author{
Robert J. HANSEN, Ralph C. LitTLE \\ AND Peter C. FORAME \\ U. S. Naval Research Laboratory, Washington, D.C., U.S.A.
}

\begin{abstract}
Experimental and theoretical studies of early turbulence in pipe flows of drag-reducing polymer solutions are described in this work. The experiments show that a critical onset wall shear stress, which increases with solvent viscosity, is associated with the phenomenon. The relationship of the onset condition to polymer concentration is a strong function of viscosity over the range of variables considered. A theoretical, hydrodynamic stability analysis of the laminar pipe flow of a convected Maxwell fluid is presented. The results suggest that early turbulence is a hydrodynamic instability phenomenon.
\end{abstract}

\section{Introduction}

The pipe flow of very dilute polymer solutions has been studied extensively during the past decade. Most investigators have reported that, for sufficiently high flow rate of the solution and molecular weight of the dissolved polymer additive, the turbulent flow drag of the polymer solution is less than that for a Newtonian fluid of the same density and steady-shear viscosity. Recently, the present authors ${ }^{5)}$ and others ${ }^{12,16)}$ have also observed departures from Newtonian behavior in pipe flows of these solutions at subcritical Reynolds numbers; i.e., for $U R \rho / \mu$ less than 2300, the approximate value at which the laminar-to-turbulent transition occurs in a Newtonian fluid. Here $U$ denotes the maximum flow velocity in the pipe, $R$ the radius, $\rho$ the solution density, and $\mu$ its viscosity. (The solutions considered subsequently are sufficiently dilute that no distinction is necessary between solvent and solution density or viscosity.) In the present work recent experimental results relating to this phenomenon, which shall be termed early turbulence, are summarized. Theoretical evidence which suggests that early turbulence is a hydrodynamic instability phenomenon is then presented.

\section{Experimental Work}

Early turbulence is peculiar to flows where high shear stresses are realized at subcritical Reynolds

\footnotetext{
* Received on February 16, 1973

Presented at First Pacific Chemical Engineering Congress, Kyoto, Oct. 11-14, 1972

R. J. Hansen, U. S. Naval Research Laboratory, Washington, D. C. 20375, U. S. A.
}

numbers. Examples are flows of aqueous solutions in capillary tubes ${ }^{12,16)}$ and flows in larger pipes of solutions made up with more viscous solvents ${ }^{5}$. The necessity for large stresses is due to the fact that a welldefined onset wall shear stress $\tau *$ must be exceeded before early turbulence can be observed. This is apparent in Fig. 1, which is a shear stress vs. flow rate plot for a polyethylene oxide solution flowing in a $0.660 \mathrm{~cm}$ diameter pipe. The solvent is a waterglycerine mixture of viscosity 0.0580 poise, and the average molecular weight of the polymer is $9 \times 10^{5}$. The Reynolds number at the onset point is 1440 in this case. (Details of the experimental apparatus and procedures used to obtain this plot and those presented subsequently have been presented elsewhere ${ }^{5)}$.) At wall shear stresses below $\tau^{*}$ the flow of the polymer

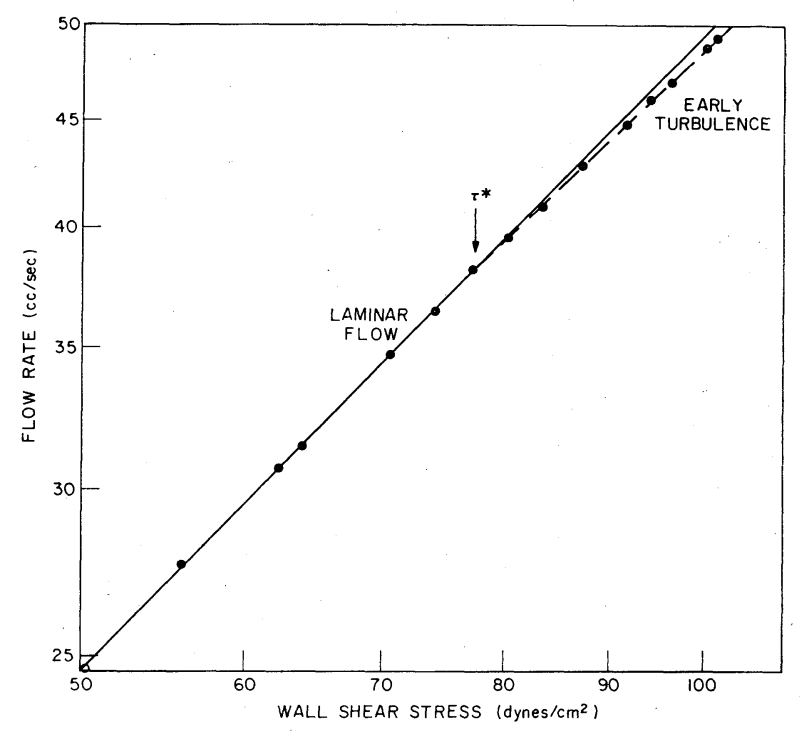

Fig. 1 An example of early turbulence in a $0.660 \mathrm{~cm}$ diameter pipe 


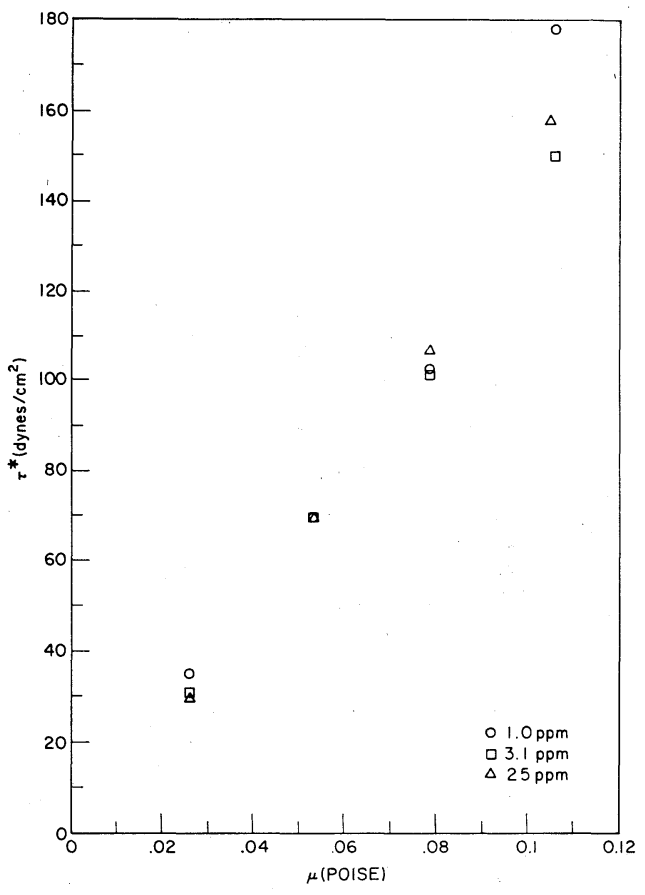

Fig. 2 The effect of solvent viscosity on the onset wall shear stress in a $0.553 \mathrm{~cm}$ diameter pipe

solution is seen to be Newtonian. When the wall shear stress exceeds $\tau^{*}$, however, the drag exerted by the polymer solution exceeds that for a Newtonian fluid flowing at the same rate. A gradual increase in the flow rate of the polymer solution would further show ${ }^{12}$ a continuous transition from early turbulence to turbulent flow with reduced drag. In contrast a Newtonian fluid exhibits an abrupt laminar-to turbulent transition at the critical Reynolds number.

To elucidate the mechanism of early turbulence, experiments have been conducted ${ }^{5)}$ to determine the variation of $\tau^{*}$ with solvent viscosity and polymer concentration. An example of the solvent viscosity dependence observed to date is shown in Fig. 2. (The polymer in this case is a polyethylene oxide condensate of mean molecular weight $7 \times 10^{6}$ and the pipe diameter $0.553 \mathrm{~cm}$. The solvent is a water-glycerine mixture, the viscosity of which is varied by changing the glycerine content.) The nearly linear relationship between $\tau^{*}$ and solvent viscosity indicates that the onset wall shear rate $S^{*}$ is a weak function of solvent viscosity over the range of variables tested. That is, the relationship between wall shear stress and wall shear rate in laminar pipe flow is

$$
\tau^{*}=\mu S^{*}
$$

The relationship of $\tau^{*}$ to polymer concentration observed with this same polymer, solvent, and pipe flow apparatus, is shown in Fig. 3. At the lowest solvent viscosity $\tau^{*}$ is essentially independent of polymer concentration. A variation in $\tau^{*}$ with concentration becomes apparent as solvent viscosity is increased, however; and the minimum in the $\tau^{*}-\ln$ (concentration) curve resembles that reported previously ${ }^{12)}$ for early

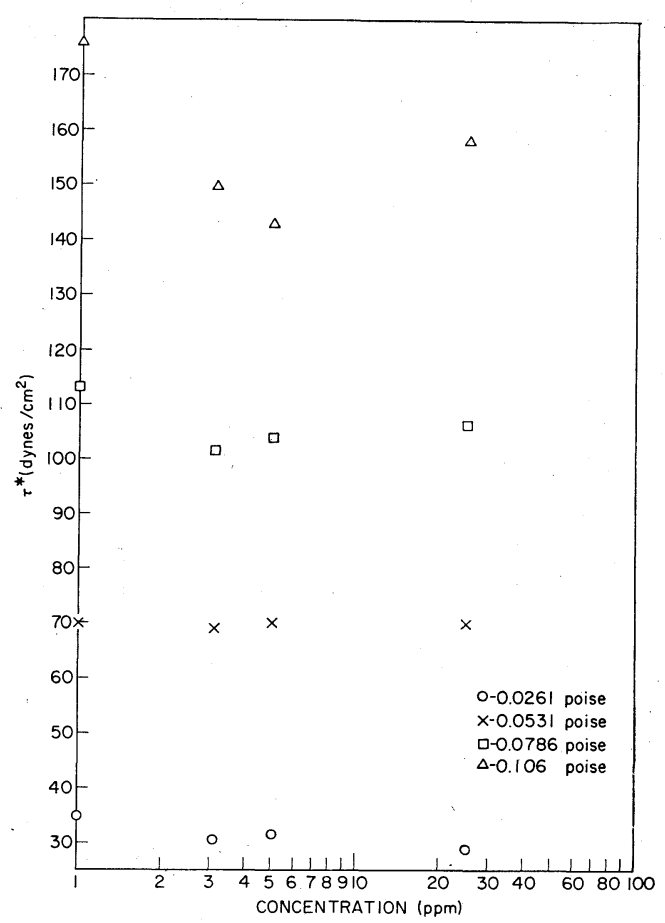

Fig. 3 The polymer concentration dependence of onset wall shear stress in a $0.553 \mathrm{~cm}$ diameter pipe

turbulence in the capillary tube flows of aqueous polyethylene oxide solutions. The data of Fig. 3 indicate that the formation of the minimum in the $\tau^{*}-\ln$ (concentration) relationship is a strong function of viscosity. An additional factor in causing the decrease in $\tau^{*}$ with increasing concentration at the highest viscosity and lowest concentrations may be mechanical degradation of the polymer molecules in the flow. Mechanical degradation does not account for the increase in $\tau^{*}$ with concentration above the 3 to $5 \mathrm{ppm}$ concentration range, however, nor for the fact that the increase becomes more apparent with increasing viscosity.

Early turbulence is not an entrance effect, since it was clearly observable at both 230 and 745 diameters from the upstream end of the $0.660 \mathrm{~cm}$ pipe. The latter distance is much larger than that ordinarly associated with entrance effects. Moreover, the value of $\tau^{*}$ was measured at both locations for one value of solvent viscosity, polymer concentration, and molecular weight; and the results agreed within the experimental uncertainty of the measurements $( \pm 5 \%)$.

\section{Theoretical Work}

The suggestion has been made ${ }^{5)}$ that early turbulence is a hydrodynamic instability phenomenon. That is to say, the presence of the polymer additive may change the minimum Reynolds number at which small disturbances to the laminar flow of a fluid are amplified, rather than damped by viscous effects. Such an explanation seems plausible in the light of experiments ${ }^{3}$ ) showing that the stability of laminar flows 
between rotating, concentric cylinders can be affected by a polymer additive. Moreover, a significant body of theoretical work has been conducted in an attempt to ascertain the effects of viscoelasticity (which may be imparted to a Newtonian fluid by the introduction of a polymer additive) on laminar flows in a variety of geometries. For example an approximate analysis has been given ${ }^{4)}$ of the stability in pipe flow of a so-called "Maxwell fluid", described by the following constitutive equation:

$$
\left(1+\lambda \frac{\partial}{\partial t}\right)\left(\tau^{i j}+p \delta^{i j}\right)=\frac{\partial v^{i}}{\partial x^{j}}+\frac{\partial v^{j}}{\partial x^{i}}
$$

Here $p$ and $\tau^{i j}$ are the pressure and the $i j$-th component of the stress tensor, respectively, both normalized by dividing by $\mu U / R$, where $R$ denotes the tube radius. The symbol $\lambda$ denotes a normalized relaxation time $T U / R$, where $T$ is the relaxation time of the fluid. $v^{i}, x^{i}$, and $t$ denote the $i$-th velocity component, distance coordinate, and time, respectively, all normalized with respect to $U$ and/or $R . \delta^{i j}$ is the Kronecker delta function. The elastic effect, represented by the time derivative term in Eq.(2), was found to lower the critical Reynolds number. Similar predictions have been obtained ${ }^{1)}$ in studies of the flow of a Maxwell fluid between parallel plates. These results appear consistent with the view that early turbulence is related to hydrodynamic stability considerations.

Conclusions drawn on the basis of previous theoretical work about the relationship of early turbulence and hydrodynamic stability are open to question for at least two reasons, however. The first is that the method of analysis used previously ${ }^{4)}$ in studying the pipe flow stability of a Maxwell fluid may not have been satisfactory. Only the first two terms of a series expansion for the solution to the problem were retained in the Galerkin method; and the convergence of this twoterm approximation to the exact solution was not demonstrated by examining higher order terms in the series. Second, the applicability of Eq.(2) to polymer solutions has not been established. Therefore, it is not clear a priori that analyses based on this equation are pertinent to early turbulence.

To further elucidate the explanation for early turbulence, a theoretical study of the pipe flow stability of a convected Maxwell fluid ${ }^{19)}$ has been conducted. The constitutive equation for this fluid is

$$
\begin{aligned}
\tau^{\prime i j}= & {\left[v^{i},{ }_{m} g^{m j}+v^{j},{ }_{m} g^{i m}\right]-\lambda_{c}\left\{\frac{\partial \tau^{\prime i j}}{\partial t}\right.} \\
& \left.+\tau^{\prime i j},{ }_{m} v^{m}-v^{i},{ }_{m} \tau^{\prime m j}-v^{j},{ }_{m} \tau^{\prime i m}\right\}
\end{aligned}
$$

where

$$
\tau^{i j} p g^{i j}+\tau^{i j}
$$

Here $g^{i j}$ denotes the conjugate metric tensor and $v^{i}{ }_{m}$ the covariant derivative of $v^{i}$. All velocities, stresses, and the pressure are normalized in the same manner as in Eq.(2), as is the (normalized) relaxation time $\lambda_{c}$ associated with this constitutive relationship. In this case the elastic effect is represented by the sum of terms multiplying $\lambda_{c}$. This relationship was chosen because it is known to provide a qualitatively correct rheological description of polymer solutions in a variety of circumstances ${ }^{14)}$.

The stability analysis consisted of the following steps. (a) The continuity and momentum equations were obtained in cylindrical coordinates for a convected Maxwell fluid. (b) Following the procedure used in previous stability analyses of Newtonian fluids ${ }^{2,11,18)}$, small, axisymmetric perturbations of velocities, stresses, and pressure from their values in steady, laminar, pipe flow were introduced. The linear, partial differential equations for the perturbation quantities were obtained as functions of $t, r$, and $z$, where $r$ and $z$ denote the (normalized) distances from and parallel to the tube axis, respectively. (c) Each perturbation quantity was assumed to be of the form

$$
q=\operatorname{Real}\{\hat{q}(r) \exp [i \alpha(z-c t)]\}
$$

where $\hat{q}(r)$ represents the complex magnitude of the quantity, $\alpha$ the (normalized) wavenumber of the perturbation, and $c$ the (normalized) phase velocity. ( $\alpha$ was considered a real quantity and $c$ complex.) The expressions of the form of Eq.(4) were substituted into the partial differential equations for the perturbation quantities, thereby reducing them to ordinary differential equations for the complex perturbation magnitudes. (d) Consideration of the orders of magnitude of the various terms in these equations suggested that Eq.(2) is a good approximation to Eqs.(3) in axisymmetric flows for the special case of $|c| \gg 1$. That is, the dominant elastic term of Eq.(3a) appeared to be the time derivative of $\tau^{\prime i j}$ for axisymmetric perturbations of sufficiently large phase velocities. (e) The stability problem was solved by assuming that $|c|$ and Reynolds number $N$ were large compared to unity and that all other elastic terms in Eq. (3a) were negligible compared to that containing the time derivative of $\tau^{i j}$. Details of this solution are given in the Appendix. (f) The solution obtained on this basis was substituted back into the equations where all elastic terms had been retained. This step confirmed the dominance of the time derivative term in Eq.(3a) over the other elastic terms for large $|c|$ and $N$.

The equation for $\hat{\Phi}_{n}(r)$, the complex magnitude of the $n$-th eigensolution for the perturbation stream function, obtained from the analysis was

$$
\hat{\Phi}_{n}(r)=J_{1}(i \alpha r)-\frac{J_{1}(i \alpha) J_{1}\left[\left(n+\frac{3}{4}\right) \pi r\right]}{J_{1}\left[\left(n+\frac{3}{4}\right) \pi\right]}
$$

Here $n$ may be any large positive integer and $J_{1}$ denotes the Bessel function of the first kind and of order one. An identical result was obtained previously by Schensted $^{18)}$ for a Newtonian fluid. The associated expression for $\alpha c$ obtained in the present analysis was 


$$
\alpha c=-\frac{i}{2 \lambda_{c}}+\frac{i}{2 \lambda_{c}} \sqrt{1-\frac{4 \lambda_{c}}{N}\left(n+\frac{3}{4}\right)^{2} \pi^{2}}
$$

For the case of $\lambda_{c}=0$ (Newtonian fluid) a series expansion of the square root term in Eq.(6) shows that

$$
\operatorname{limit}_{\lambda_{c} \rightarrow 0} \alpha c=-\frac{i\left(n+\frac{3}{4}\right)^{2} \pi^{2}}{N}
$$

This is also identical to the result obtained previously in Schensted's analysis ${ }^{18)}$ of the Newtonian case.

These results are significant in a number of respects. First, the critical Reynolds number for the pipe flow of a convected Maxwell fluid cannot be ascertained solely by consideration of axisymmetric disturbances with large phase velocities. The imaginary part of the product $\alpha c$ from Eq.(6) is negative for all values of $\lambda_{c}$, $N$, and $n$. Consequently, all disturbances treated in the analysis decay with time, a result which follows from substitution of Eq.(6) into Eq.(4). A number of previous investigators ${ }^{2,15,18)}$ have drawn the same conclusion about pipe flows of Newtonian fluids, and it is also apparent from Eq.(7) of the present analysis. Prediction of the critical Reynolds number of pipe flows apparently requires more elaborate analysis in which lower phase velocity and/or three dimensional disturbances are considered.

The present analysis is nevertheless germane to the early turbulence phenomenon. It shows that the rate of damping of the disturbances considered is affected by the elastic character of the fluid. The ratio I of the imaginary part of $\alpha c$ from Eq.(6) to that from Eq.(7) satisfies the following condition:

$$
I \leqslant \frac{N}{\lambda_{c}} \frac{1}{2\left(n+\frac{3}{4}\right)^{2} \pi^{2}}
$$

The inequality applies when

$$
\frac{\lambda_{c}}{N}\left(n+\frac{3}{4}\right)^{2} \pi^{2}
$$

is less than $1 / 4$ and the equality when it is equal to or greater than $1 / 4$. The present analysis is valid when

$$
\left(n+\frac{3}{4}\right)^{2} \pi^{2} / N
$$

is very large compared to unity. Under these circumstances Eq.(8) shows that $I<1$ when $\lambda_{c}$ exceeds some value much less than unity. Thus, axisymmetric disturbances with large phase velocities are not as quickly damped by viscous effects in the convected Maxwell fluid as in the Newtonian one, for sufficiently large $\lambda_{c}$. Elastic effects exert a destabilizing influence on the disturbances considered as $\lambda_{c}$ is increased to a large enough value, in other words. (The same conclusion follows from a different type of mathematical analysis of the problem than that outlined above ${ }^{8}$.) It appears quite likely, in light of this result, that early turbulence is a consequence of a similar destabilizing effect of elastic forces on the lower phase velocity or nonaxisymmetric perturbations not considered in the analysis.

This view of early turbulence is also substantiated by comparing the conditions which Eq.(8) indicates are conducive to destabilization with the experimentally observed characteristics of the phenomenon. The value of I depends on the ratio of $N$ to $\lambda_{c}$, which in terms of dimensional quantities is $R^{2} \rho / T_{c} \mu$. Here $T_{c}$ denotes the (dimensional) relaxation time associated with the convected Maxwell model. Experimental and theoretical work available to date ${ }^{9,10,13)}$ indicate that $T_{c}$ increases with polymer concentration (in dilute solutions) and molecular weight, as well as solvent viscosity. Thus, the analysis predicts that the degree of destabilization increases (I decreases) with each of these variables, as well as with decreasing tube radius. The experimental results presented above, together with those of Little and Weigard ${ }^{12)}$, show that large molecular weights and solvent viscosities and small tube radii are also conducive to the observation of early turbulence in pipe flow systems. Additionally, increasing polymer concentration promotes early turbulence in sufficiently dilute solutions.

While the experimental and theoretical work presented above are indicative that early turbulence is related to the behavior of small perturbations to the flow, more research is needed to establish this point rigorously. Experiments to determine the timeaveraged velocity profile and the nature of any fluctuating velocity components in the early turbulence regime are recommended. Theoretical work should be extended to lower phase velocities in an attempt to identify an unstable regime for axisymmetric perturbations. Similar theoretical studies based on more complicated, but more realistic, constitutive equations may also be in order. Investigation of nonaxisymmetric perturbations would be desirable, but the mathematical difficulties associated with such analyses for Newtonian fluids $^{6)}$ suggest that investigations of this type are not feasible at present.

\section{Conclusions}

Early turbulence can be observed in flows of dragreducing polymer solutions through pipes, when the solvent viscosity is sufficiently large and the pipe diameter sufficiently small to cause large shear stresses at subcritical Reynolds numbers. The onset wall shear stress for the phenomenon increases almost linearly with solvent viscosity for a given polymer concentration and molecular weight over the range of variables tested. Its relationship to concentration is a strong function of solvent viscosity. The results of new theoretical work on the stability of high-phase-velocity, axisymmetric perturbations in laminar pipe flow of a convected Maxwell fluid have been presented. They substantiate the view that early turbulence is a hydrodynamic instability phenomenon. 


\section{Appendix}

Steps (a) through (d) of the mathematical analysis described in the text; together with the assumption that the dominant elastic term in Eq.(3) is the time derivative of $\tau^{\prime i j}$, reduce the stability problem to one of solving the differential equation

$$
i \alpha\left(1-r^{2}-c\right) y=\frac{1}{N_{c}}\left(L^{2}-\alpha^{2}\right) y
$$

where

$$
\begin{aligned}
& y=\left(L^{2}-\alpha^{2}\right) \hat{\Phi} \\
& L^{2}=\frac{d^{2}}{d r^{2}}+\frac{1}{r} \frac{d}{d r}-\frac{1}{r^{2}}
\end{aligned}
$$

and

$$
N_{c}=N\left(1-i \alpha c \lambda_{c}\right)
$$

The boundary conditions are

$$
\hat{\Phi}(0)=\text { finite } ; \quad \hat{\Phi}(1)=\hat{\Phi}^{\prime}(1)=0
$$

where the prime denotes differentiation with respect to $r$. These conditions express the facts that the fluid velocity is finite at the tube centerline and zero at the wall. For large $|c|$ the quantity $\left(1-r^{2}\right)$ in Eq. (9a) may be neglected (as can be justified rigorously by use of series expansions for confluent hypergeometric functions ${ }^{17)}$.) Eqs.(9a) and (9d) are then combined to give

$$
\left(L^{2}-\alpha^{2}\right)\left(L^{2}+\beta^{2}\right) \hat{\Phi}=0
$$

where

$$
\beta^{2}=i \alpha c N_{c}
$$

The solution to Eq.(11) which satisifies the boundary condition at $r=0$ has the form

$$
\Phi=C_{1} J_{1}(i \alpha r)+C_{2} J_{1}(\beta r)
$$

Additionally, the boundary conditions at the wall necessitate that

or more simply $\left|\begin{array}{ll}J_{1}(i \alpha) & J_{1}(\beta) \\ J_{1}^{\prime}(i \alpha) & J_{1}^{\prime}(\beta)\end{array}\right|=0$

$$
\frac{\beta J_{0}(\beta)}{J_{1}(\beta)}=\frac{i \alpha J_{0}(i \alpha)}{J_{1}(i \alpha)}
$$

The asymptotic behavior of the Bessel function of the first kind and of order $\nu$ for large $|\beta|$ is 7 )

$$
J_{\nu}(\beta) \sim \sqrt{\frac{2}{\pi \beta}}\left\{\cos \left(\beta-\frac{\nu \pi}{2}-\frac{\pi}{4}\right)\right\}
$$

Substitution of Eq.(15) into Eq.(14) shows that for large $|\beta|$ (i. e., large $N$ ),

$$
\cos \left(\beta-\frac{\pi}{4}\right) \sim 0
$$

Thus,

$$
\beta \sim\left(n+\frac{3}{4}\right) \pi
$$

The value of $\alpha c$ given by Eq.(6) is then obtained by solving Eq. (17) for $\alpha c$ and imposing the requirement that the result approach that for a Newtonian fluid as $\lambda_{c}$ approaches zero.

\section{Nomenclature*}

$$
\begin{array}{ll}
c & =\text { (normalized) phase velocity of a perturbation } \\
C_{1}, C_{2} & =\text { constants } \\
g^{i j} & =\text { conjugate metric tensor } \\
I & =\text { ratio of the imaginary part of } c \text { for the }
\end{array}
$$

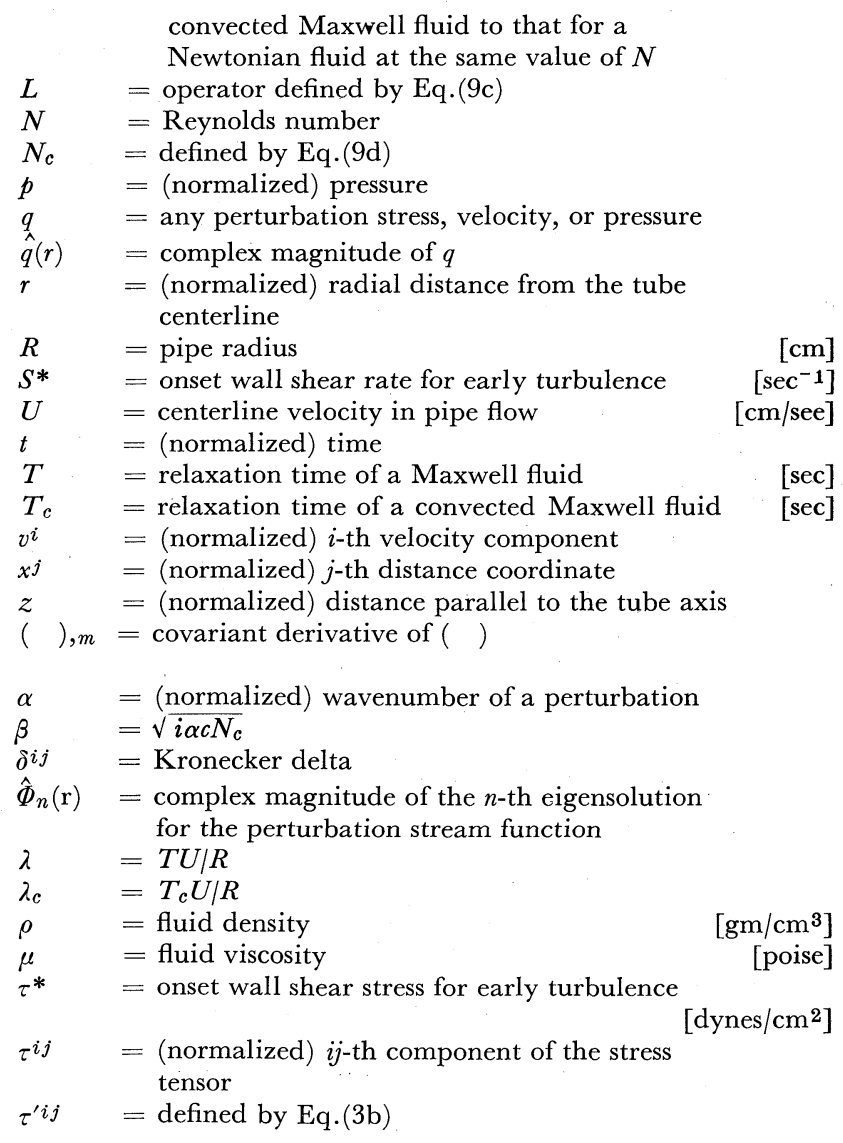

\section{Literature Gited}

1) Betchov, R. and W. O. Criminale, Jr.: "Stability of Parallel Flows", Academic Press, New York (1967)

2) Corcos, G. M. and J. R. Sellars: J. Fluid Mech., 5, 97 (1959)

3) Denn, M. M. and J. J. Roisman: AIChE J., 15, 454 (1969)

4) Derman, D.: Israel J. Tech., 5, 32 (1967)

5) Forame, P. C., R. J. Hansen and R. C. Little: AIChE J., 18, 213 (1972)

6) Graebel, W. P.: J. Fluid Mech., 43, 279 (1970)

7) "Handbook of Mathematical Functions", M. Abramowitz and I. A. Stegun, eds., Dover, New York (1965)

8) Hansen, R. J.: AIChE J., 19, 298 (1973)

9) Hansen, R. J. and R. C. Little: Chem. Eng. Progr. Symp. Ser. No. 67, 67, 93 (1971)

10) Kusamisu, S., L. A. Holmers, A. A. Moore and J. D. Ferry: Trans. Soc. Rheology, 12, 559 (1968)

11) Lin, C. C.: "The Theory of Hydrodynamic Stability", Cambridge University Press, Cambridge (1955)

12) Little, R. C. and M. Wiegard: J. Appl. Polymer Sci., 14, 409 (1970)

13) Meister, B. J. and R. D. Biggs: AIChE J., 15, 643 (1969)

14) Metzner, A. B., E. A. Uebler and G. F. Chan Man Fong: AIChE J., 15, 750 (1969)

15) Pekeris, C. L.: Proc. Nat. Acad. Sci., 34, 285 (1948)

16) Ram, A. and A. Tamir: J. Appl. Polymer Sci., 8, 2751 (1964)

17) Romanovskaya, I. L. and N. M. Khalfina: U. S. S. R. Comp. Math. Phys., 7, 661 (1967)

18) Schensted, I. V.: "Contributions to the Theory of Hydrodynamic Stability", Technical Report of the College of Engineering, University of Michigan, Ann Arbor (1960)

19) White, J. L. and A. B. Metzner: J. Appl. Polymer Sci., 7, 1867 (1963)

\footnotetext{
* Normalized quantities are obtained by dividing the corresponding physical quantities by $U, R, U / R$, or $\mu U / R$.
} 